\title{
Maritime Automated Targets Recognition Algorithm Test Bed for High Resolution ISAR Imagery
}

\author{
Patrick L. Cross, PhD. \\ Raytheon Missile Systems \\ Huntsville, AL, USA
}

\begin{abstract}
A maritime automatic target recognition system is under development to perform ship classification using images from inverse synthetic aperture radar (ISAR) systems. This work will describe the algorithm framework for feature extraction, classification, and aim-point recognition. ISAR systems produce two-dimensional images of ships from the periodic motion inherent to all maritime objects and can be used to distinguish objects of interest for Homeland Security. There is a need for real-time classification of these objects. This work extends the state of the art in two ways: One - current maritime classification focuses on satellite ISAR, creating latency for classification[1]; and Two work with Homeland Security ISAR radar systems is focused on concealed weapon detection [2,3]. This work uses localized ISAR images from stationary radars to classify maritime objects. Since the aspect and cross-range scale factor are continually changing, ISAR images form distinct representations unique to each object under observation. These distinct representations are analogous to visual images in many ways. They have unique shapes and areas of strong return, much in the same way objects in passive images have unique sizes and colors. However, ISAR images are synthetic portrayals of scattered field data from active sensor RF sensors whereas visual images are observations of passive sensors. This distinction must be a considered as a design constraint in the classification system - each feature employed must be exhaustively examined regarding its physical counterpart. Within that constraint, ISAR synthetic imagery feature extraction techniques can leverage from previous work developed for passive sensors. This is especially the case of High Range Resolution (HRR) ISAR systems. After extraction from ISAR prototype images, a set of representative features will be used to train a support vector machine (SVM) classifier, a supervised learning model capable of pattern recognition.
\end{abstract}

Keywords - Inverse synthetic aperture radar, high resolution, automated target recognition

\section{INTRODUCTION}

The automated recognition of maritime targets based on inverse synthetic aperture radar systems (ISAR) has many applications including weapon systems and homeland security surveillance. Previous systems have been investigated [1]-[8]. While, Synthetic Aperture Radar (SAR) assumes stationary targets viewed by travelling radars, ISAR is the reverse. While both systems analyze differences in Doppler due to relative motions, the unknown motions of the target add a level of complexity for ISAR target recognition, especially in maritime environments [4]. The range - range rate (Doppler) technique is used to process the received data and large bandwidth coded signals achieves high resolution in the range coordinate and coherent integration of echoes collected at different viewing angles are used to achieve high cross-range resolution.

In this paper, we focus on the classification ISAR images of maritime targets exhibiting various motions with respect to the radar in a variety of sea states. An advantageous feature of maritime ISAR is, while the velocity of the target may be relative slow, the consistent pitch and roll motion due to external ocean forces intensifies the Doppler signal revealing physical features, such as mast and other superstructure forms. Another feature of maritime images is the ever present whitewater; whitecaps, propeller wash, and bow wakes. Whitewater can either help or hinder the classification of maritime objects. On one hand, bow wakes greatly aid in the detection and other phases of automated recognition [7-9], i.e. given a wake there must be a maritime object. Conversely, whitewater can distort the resultant ISAR images leading to misclassification [10]. This research has developed an effective means of whitewater detection and mitigation.

In order to classify maritime targets each image must be processed by a sequence of algorithms [4]. This research, while building upon previous work, deviates through expansion of the proposed features sets and more in-depth range profiling. Referring to Fig. 1, the following parameterized functions are user-selectable in the algorithm chain:

- $\quad$ Preprocessing

o Set the image threshold;

○ Blur the image; and

o Identify and mitigate streaks.

- Segmentation

○ Connect ship regions;

○ Identify and mitigate whitewater; and

○ Calculate the ship mid-line.

- Image transforms

○ Apply affine transforms;

○ Split the image about mid-line; and

○ Define ship sections (bow, mid, stern)

- Extract features for classification or training purposes 

○ Calculate geometric features (length, area, etc.);
o Identify wakes; and
○ Profile Doppler returns.

- Extract features for classification or training purposes

o Calculate geometric features (length, area, etc.);

○ Identify wakes; and

○ Profile Doppler returns.

- Classification

o Calculate geometric features (length, area, etc.);

○ Identify wakes; and

○ Profile Doppler returns.

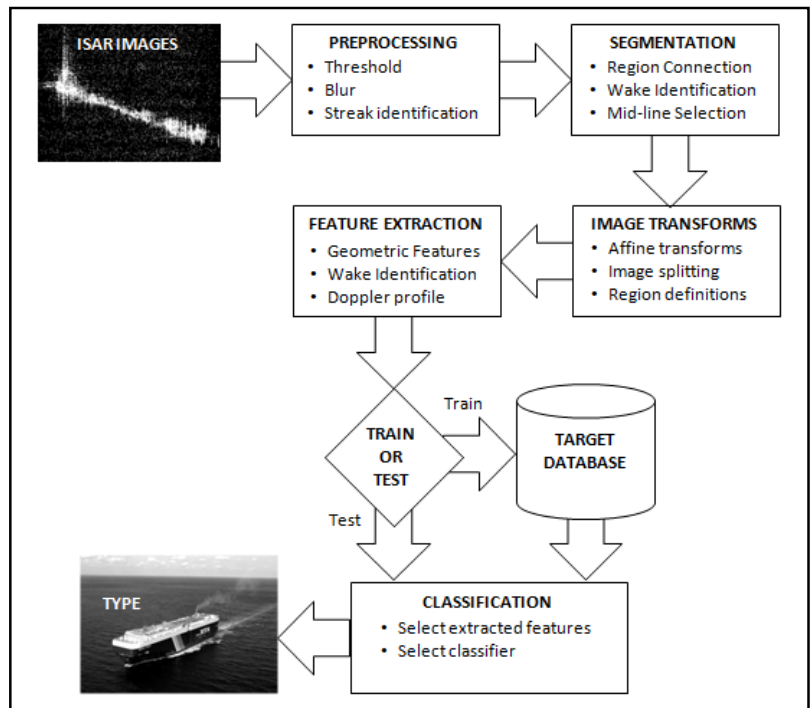

Fig. 1. Data flow diagram of algorithm chain.

The ship ISAR image is preprocessed producing an image that then can be segmented into regions yielding a hard body representation as well as wake locations. The hard-body and wake segments are ready for applicable image transformations. The selectable transforms are the final image enhancements prior to feature extraction. The features extracted are based on geometric properties (length, area, etc.) wake identification; and range profiling. The features are then used to either establish a training database or be used to classify objects using models in said database.

\section{Methodology}

The input to the overall automated recognition algorithm chain is a sequence of ISAR image with each image portrayed is in the slant-range and Doppler-frequency domains. The original image (Fig. 2) while information rich needs preprocessing to augment desirable features (i.e. hull shape, mast locations, and spinning antenna returns) while abating other effects (i.e. speckle-like noise, white water and wake returns). The next subsections will discuss the technical approach and justification for key algorithms.

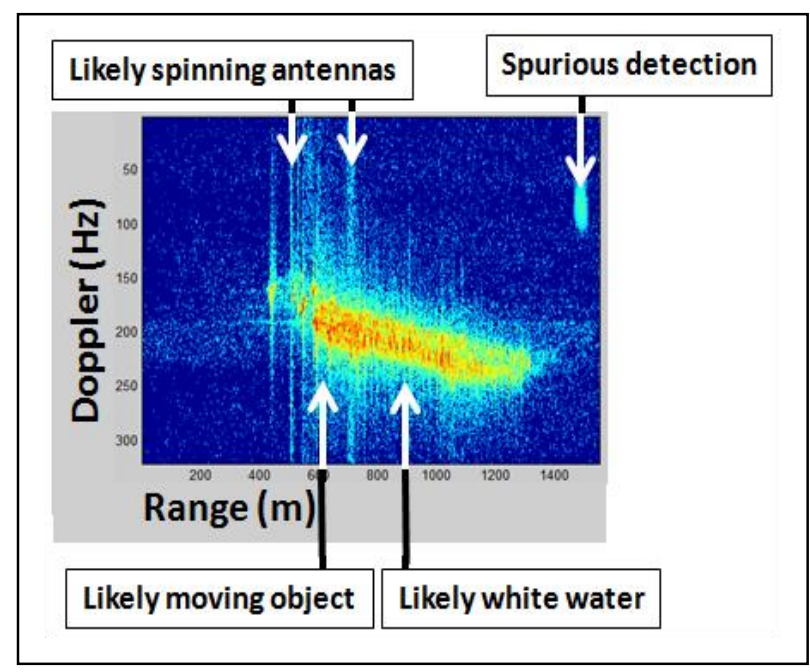

Fig. 2. ISAR image highlighting areas of concern.

\section{A. Detect and Mitigate Vertical Streaks}

ISAR imagery usually contains vertical streaks as seen in Fig. 2. These vertical streaks, several pixels wide, originate from either internal motion on the ship (e.g., spinning antennas) or multiple-bounce scattering from white water returns have adverse effects on hard-body segmentation [5]. MATRAT reduces the streaks be removing the mean or median value from each range bin. The removed streaks are then set aside for further feature extraction (Note: this is a future improvement still under design). Figure 3 depicts streak reduction in while retaining the overall range profile. The resulting image is available for segmentation

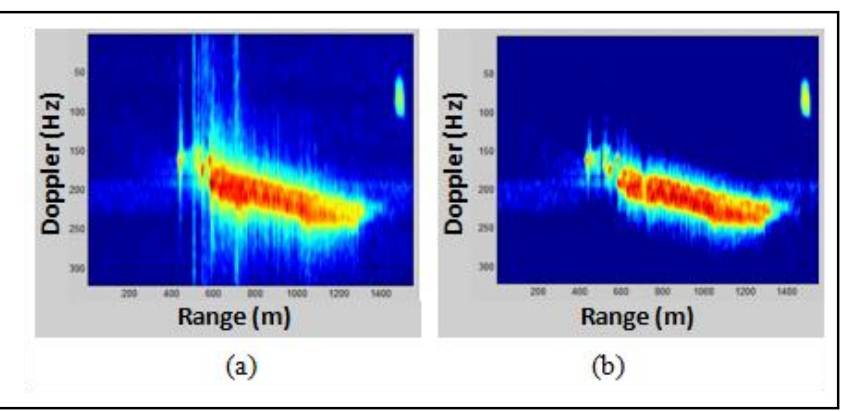

Fig. 3. Reducing streaking of ISAR image. (a) input image; (b) image with reduced streaking

\section{B. Initial Segmentation}

Segmentation partitions the image into multiple segments in order to more easily identify regions of relevance in the ISAR image (i.e. background, hull, or wakes, etc.). Edges in an ISAR scene are caused by changes physical properties of the scene, such as illuminating power, aspect angle, distance and radar cross section, etc. Therefore, direct relationships exist between the edges and the physical properties of the scene, and critical information can be recovered from the edge 
image [13]. The MATRAT system uses the MATLAB

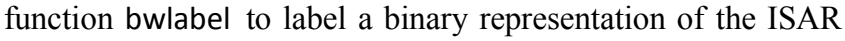
image [26]. The general procedure outlined in [14] is as follows:

a. Run-length encodes the input image;

b. Scan the runs, assign preliminary labels and recording label equivalences in an equivalence table;

c. Resolve the equivalence classes; and

d. Relabel the runs based on the resolved equivalence classes.

Figure 4 demonstrates the result of initial segmentation. (a)

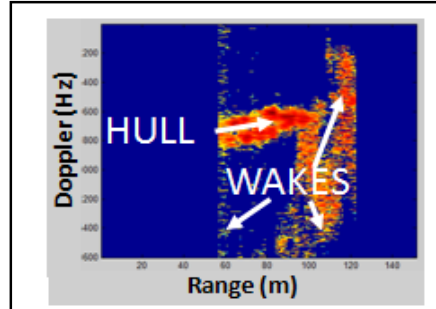

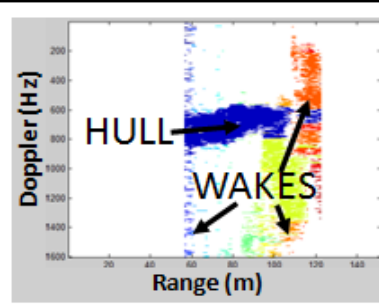

(b)
Fig. 4. Segmentation: (a) input image; (b) labeled segments

\section{Wake Identification and Mitigation}

While segmentation is a primary purpose of separating the hard-body from other regions, it sometimes necessary to apply a further wake segmentation algorithms. Figure 5 illustrates such a case; a wake is still attached to the hard-body after initial segmentation, distorting the image, and eventually, misclassification.

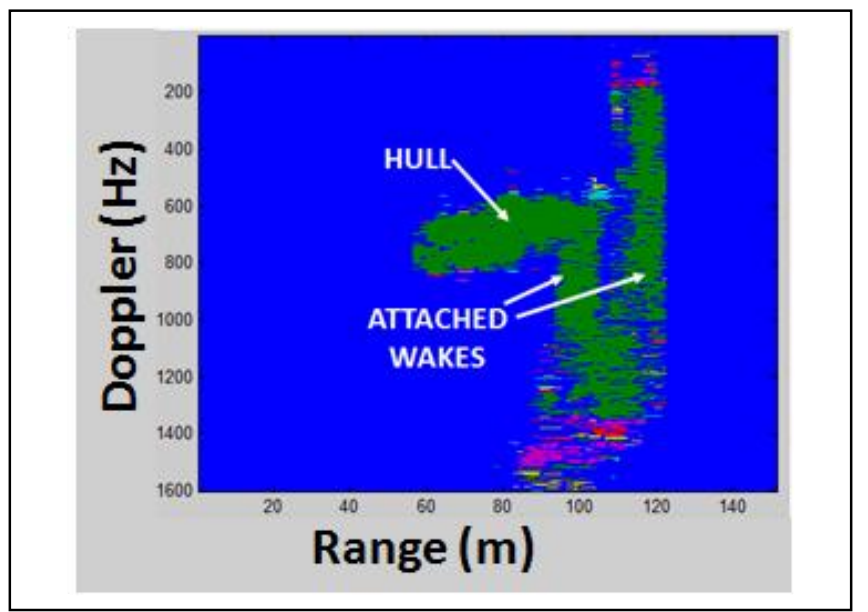

Fig. 5. Attached wakes after initial segmentation.

Attached wakes are separated from the hard-body through an image erosion technique [15]. Erosion simply 'pares' the image along the outermost path leaving the erosion outline and an image containing the hard-body and wake remnants. Figure 6 illustrates the erosion technique.

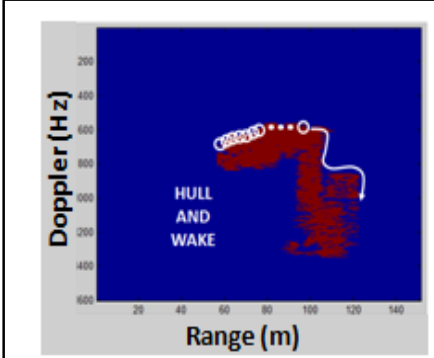

(a)

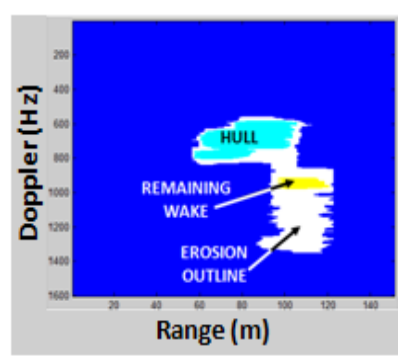

(b)
Fig. 6. Eroding the ISAR image to separate wakes from the hard-body.

The subsequent relabeling produces multiple regions and a method is needed to select the most hard-body like region. In order to facilitate selection local features are calculated for each region. Table 1 show an example feature set for a relabeled ISAR image. Typical features include:

- $\quad$ Region area, in pixels;

- Euclidian distance of the region to the image center, in pixels;

- Solidity, a scalar specifying the proportion of the pixels in the convex hull that also included in the region. The convex hull is defined as the smallest convex polygon that can contain the region;

- The maximum, minimum and sum of intensities of the region;

TABLE I. REGION STATISTICS

\begin{tabular}{|r|r|r|r|r|r|r|}
\hline Label & Area & Distance & Solidity & Max & Min & Sum \\
\hline 0 & 16482 & 206.334 & 0.414 & 4.313 & 1.402 & 16010.217 \\
\hline 1 & 216700 & 12.315 & 0.896 & 3.610 & 1.175 & 13013.108 \\
\hline 2 & 7261 & 110.914 & 0.872 & 5.729 & 2.883 & 20214.026 \\
\hline 3 & 1308 & 149.196 & 0.916 & 3.077 & 1.486 & 1507.073 \\
\hline
\end{tabular}

Rules for selection are as follows:

1. The selected region cannot have the lowest solidity ratio, eliminating the erosion outline from consideration.

2. The selected region cannot have pixels in any corners, eliminating the background. Merge all "similar" select regions. A nominal similarity, $S$, is defined as a normalized score

$$
S=\sqrt{(\operatorname{Max})^{2}+(\text { Mean })^{2}}
$$

3. Any region with a normalized score within ten percent of the highest scoring region is determined to be similar and merged with the highest scoring region. The merged region is designated as the hard-body and will dilated by the same amount as the preceding erosion (Fig 7). 


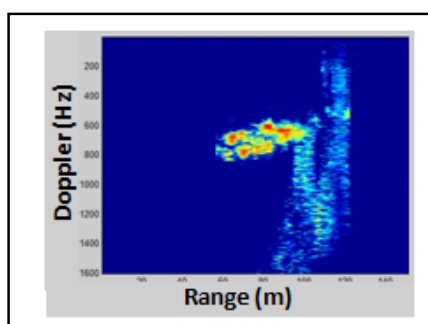

(a)

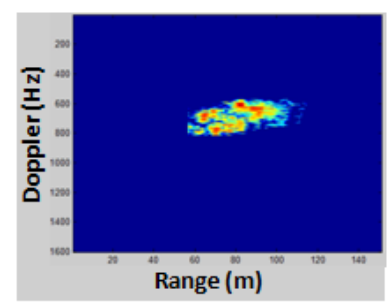

(b)
Fig. 7. Final hard-body ISAR image after segmentation, erosion and dilation: (a) original image; (b) hard-body ISAR image.

\section{Length Estimation}

True length is a very powerful discriminator between classes of maritime targets. Very accurate a priori information is available regarding most major commercial and naval vessels [18]. ISAR images can accurately measure the apparent length of maritime targets but the calculation of true length requires the ISAR/target aspect angle. MATRAT has the ability to estimate the true length. True length is computed by taking the difference in meters between the bow edge bin and the stern edge bin. This distance is then divided by the cosine of the estimated aspect angle, producing an estimate of the true length. If the aspect angle is not provided then the apparent length will be used in a relative sense, i.e., the target is the longest object in the mission. This requires contextual discrimination and is future work.

\section{E. Range Profiling}

Range profiling, in this context, is defined as Doppler returns per range bin and are a key component in the classification of the target [17]. Figure 8 shows an ISAR image and three proposed profile measures.

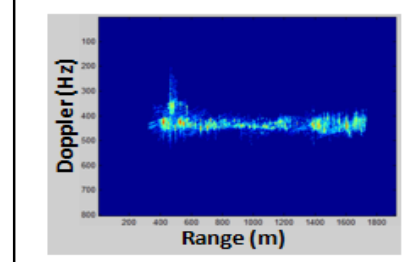

(a)

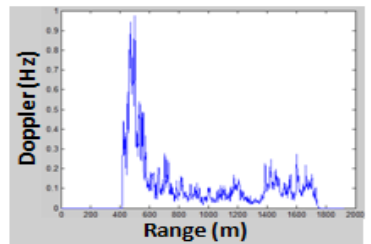

(c)

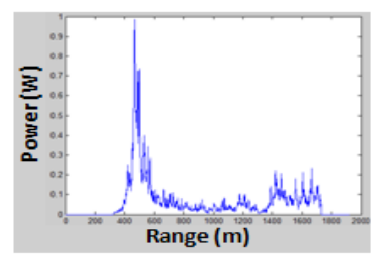

(b)

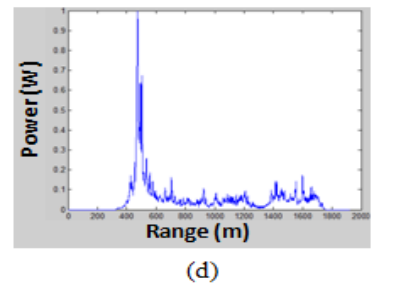

Fig. 8. Doppler image a different range profiles; (a) original image; (b) total intesity per range bin (c) extent (width) of Doppler returns by range bin; (d) the product of (b) and (c)The proposed profiles reflecting of the hard-body ship are defined as follows:

- Total Doppler intensity of each range bin - indicative of strong radar reflectors;

- The Doppler extent, also known as the Doppler width indicative of taller structures on the target; and
- The product of the total Doppler and the Doppler extent - indicative of the tallest and strongest reflectors.

MATRAT calculates these profiles per image as available features for training and classification. In addition, all features are calculated for the total ship and three other sections; bow, midship, and stern.

Current Doppler feature suites used in this evaluation are shown in Fig. 9:

- Total number of significant total intensity peaks per section;

- The distance of the peak extents from the bow edge per section

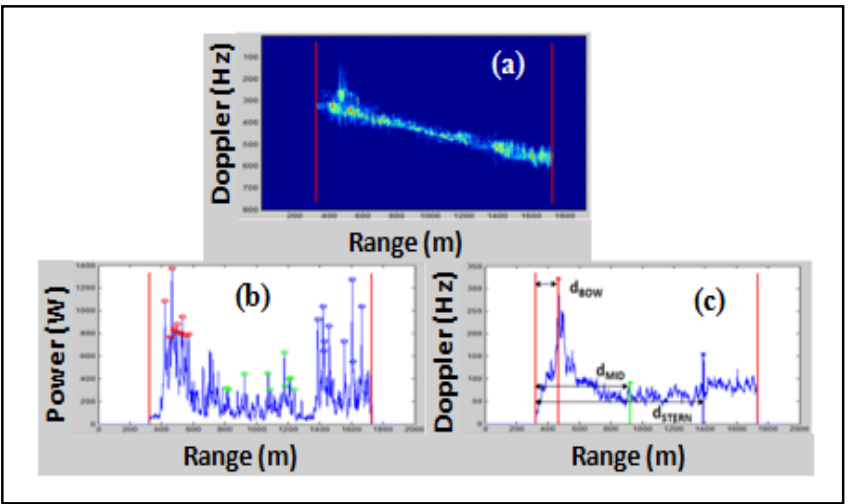

Fig. 9. Range profile features (a) original image; (b) signiificant peaks in total intensity per range bin by section (c) the distance ofrom the maximum extent to the bow edge per range bin by section.

\section{F. Features and Feature Selection}

There are many types of surface ships, both commercial and naval combatants. In general, naval ships are bulkier than merchant ships, having flowing lines and usually less deckhouse and superstructure [22]. In addition, each of the classes have specialized purposes and as such have differing image shapes. For that reason, MATRAT calculates all features by section (bow, mid, and stern). While having a rich feature set can aid in classification, care should be exercised regarding the dimensionality of the feature vector used in the classification process. The curse of dimensionality refers to problems when analyzing data in high-dimensional spaces leading to the possibility of overfitting the data, leading to incoherent classification [25]. Users of MATRAT can select from a rich feature set and quickly determine which features have the most impact to the classification and help alleviate the problem of over-fitting. Table 2 lists all available features by category and section. Currently, the feature categories are unweighted geometric features, weighted geometric features, length features, and Doppler features (Table II). 
TABLE II. FEATURES BY CATEGORY BY SHIP SECTION

\begin{tabular}{|c|c|c|}
\hline \multicolumn{3}{|c|}{ Unweighted } \\
\hline Area & Length & Perimeter \\
\hline All & All & All \\
\hline Bow & Bow & Bow \\
\hline Mid & Mid & Mid \\
\hline Stern & Stern & Stern \\
\hline
\end{tabular}

\begin{tabular}{|c|c|c|c|}
\hline \multicolumn{4}{|c|}{ Weighted } \\
\hline Centroid & $\begin{array}{c}\text { Mean } \\
\text { Intensity }\end{array}$ & $\begin{array}{c}\text { Max } \\
\text { Intensity }\end{array}$ & $\begin{array}{c}\text { Sum of } \\
\text { Intensities }\end{array}$ \\
\hline All & All & All & All \\
\hline Bow & Bow & Bow & Bow \\
\hline Mid & Mid & Mid & Mid \\
\hline Stern & Stern & Stern & Stern \\
\hline
\end{tabular}

\begin{tabular}{|c|c|}
\hline \multicolumn{2}{|c|}{ Range Profile } \\
\hline \# of Significant Peaks & Distance of Maximum Extent \\
\hline All & All \\
\hline Bow & Bow \\
\hline Mid & Mid \\
\hline Stern & Stern \\
\hline
\end{tabular}

a) Unweighted features: These are features that do not involve intensity measurements and calculated for all four sections. The current unweighted features are:

- Area is the number of image pixels;

- Perimeter is a measure of Doppler variation; and

- Length is the difference in range of the sections.

b) Weighted features: These are features that incorporate intensity measurements and calculated for all four sections. The current weighted features are:

- The maximum intensity;

- The mean intensity;

- The sum of intensities; and

- The centroid.

c) Range profile features: These are features that charcterize the Doppler measurements of the range profile. The current range profile features are:

- The number of significant peaks in the sum of intensities range profile; and

- The ratio of the distance from the largest extent to the bow edge;

\section{G. Classification}

In automated target recognition classification is the problem of identifying to which of a set of classes, an real observation belongs, on the basis of a training set of data containing observations (real or modeled) whose class membership is known. MATRAT uses a support vector machine (SVM) classifier. SVMs have already been applied in other radar target recognition problems for ground, air and maritime targets [19-21]. The support-vector is a learning machine for two-group classification problems. The machine conceptually implements the following idea: non-linearly mapped to a very high dimension feature space. In this feature space a linear decision surface is then constructed and subsequent observations are classified [25].

\section{RESUlTS}

\section{A. Current Data Sets}

Current data sets are comprised of three ships, one automotive carrier (the Auriga Leader), one cruise ship (Ms. Zandaam), and an unknown fishing vessel. All ISAR images have unknown aspect angles, but the Doppler and range bin sizes are known. While the current data sets do not pose a difficult classification problem, they were well suited for MATRAT algorithm prototype design, especially in wake and streak mitigation.

\section{B. High Fidelity Models Data set}

Due to cost and classification restrictions it is impractical to secure adequate amounts ISAR data to further develop the ATR capabilities of MATRAT. However, with the advent of faster computers it possible to improve the MATRAT by generating ISAR images from highly detailed maritime models. With these inexpensive models, we can push MATRATs capabilities especially in classification of populated maritime scenes. Current plans include the ISAR imaging of ten models of modern combatants in realistic scenarios.

\section{Introducing Random Bias and Noise into Features Extracted from Current Data Set}

While the limited data set was adequate for feature extraction, it posed no stressing scenarios regarding classification. In order to test the prototype SVM classifier in the design phase, we introduced bias and increased noise to ensure scenes in which the maritime objects had overlapping features, specifically estimated length and total number of significant peaks. Figure 10 contrasts the original scenario and the obvious separation and the scenario with additional random bias and noise.

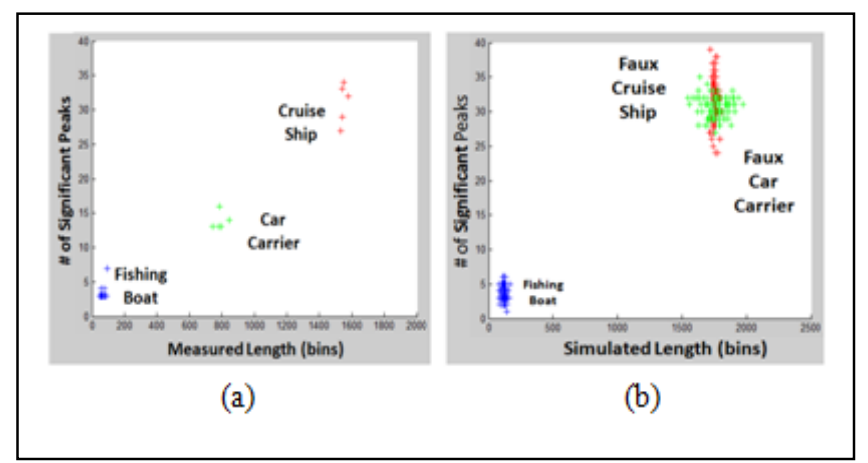

Fig. 10. ISAR 2-D Feature sets (a) original features; (b) features with additional random bias and noise. 
Another reason for choosing these features, previous work has shown good classification results using length and number of peaks as primary features [17]. While this sparse feature space has shown good results in classifying distinct classes, we believe expanding the feature sets to include sectional features. This allows us to begin to see the benefit of this approach. While many combatant ships with have similar lengths, each class of combatant ship has a specialized purpose, leading to differing range profiles. We believe the expansion to sectional features will exploit these differences.

\section{Results}

a) Two overlapping features performance: Referring to Table III, the SVM classifier was present three classes (Faux Car Carrier - CC , Faux Cruise Ship - CS, and Fishing Boat FB) and two features (Estimated Length and \# of Significant Peaks - ALL). As expected the results of the SVM classifier were not very good with each car carrier object being miscassified as a cruise shi resulting in an overall performance of Accuracy $=66.67 \%$ total classification accuracy

b) Sectional Features Performance: By replacing the total number of signicant peaks of all sections two the number of significant peaks per section the performance increases to $100 \%$ total classification accuracy.

Granted this is a straightforward example, clearly it illustrates the advantage of the sectional featuresh. We expect to see improvement in interclass classification of maritime combatants with this methodology.

\section{CONCLUSIONS}

A test bed for maritime automated ISAR image recognition has been developed, with detailed discussion of each of the current algorithm components. Special emphasis was placed on the mitigation of wakes and streaks from the ISAR images. In additional, the efficacy of expanding the features to a sectional approach was shown to have a real potential in future systems. Finally, future work was outlined to generate adequate amounts of relevant ISAR imagery using high fidelity commercial to further advance the MATRAT

\section{ACKNOWLEDGMENT}

P. L. C. thanks Ben Mitchell for his help in understanding the ISAR imagery from a physics-based point of view.

\section{REFERENCES}

[1] 1. D. Pastina, P. Lombardo, F. Fico "Detection of ship targets in COSMOSkyMed SAR images", 2011 IEEE Radar Conference, Kansas City (USA), May 2011.

[2] 2. Fernades, J., Rappaport, C.M. ; Martinez-Lorenzo, J.A. ; Hagelen, M. "Experimental results for standoff detection of concealed body-worn explosives using millimeter-wave radar and limited view ISAR processing", 2010 IEEE International Conference on Digital Object Identifier, Boston (USA), May 2009.

[3] 3. Fernades, J., Rappaport, C.M. ; Martinez-Lorenzo, J.A. ; Hagelen, M. " A comparison of experimental and modeled results of an active millimeter wave inverse synthetic aperture radar system used to perform standoff detection of person-borne improvised explosive devices.", 2012 IEEE International Conference on Geoscience and Remote Sensing Symposium (IGARSS), Munich (Germany) July 2012.

[4] S. Musman, D. Kerr,C. Bachmann, "Automatic recognition of ISAR ship images," IEEE Transactions on Aerospace and Electronic Systems, Vol. 32, No. 4, October 1996.

[5] D. Pastina C. Spina, "Multi-feature based automatic recognitionof ship targets in ISAR," IET Radar Sonar Navigation, , Vol. 3, Iss. 4, pp. 406423, 2009

[6] S. Musman, D. Kerr,C. Bachmann, "Automatic recognition of ISAR ship images," IEEE Transactions on Aerospace and Electronic Systems, Vol. 32, No. 4, October 1996.

[7] V. Zeljkovic', Q. Li, R. Vincelette, C. Tameze, F. Liu "Automatic algorithm for inverse synthetic aperture radar images recognition and classification,” IET Radar Sonar Navigation., Vol. 4, Iss. 1, pp. 96-109, 2010.

[8] M. Menon, E. Boudreau, and P. Kolodzy, "An Automatic Ship Classification System for ISAR Imagery", Lincoln Laboratory Journal, vol. 6, no. 2, pp. 289-308, 1993.

[9] G. Zilman, A. Zapolski, and M. Marom "The Speed and Beam of a Ship From Its Wake's SAR Images," IEEE Transactions on Geoscience and Remote Sensing, vol. 42, no. 10, pp. 2335-2343, October 2004.

[10] P. Vachon "Ship Detection in Synthetic Aperture Radar Imagery," Proceedings OceanSAR 2006 - Third Workshop on Coastal and Marine Applications of SAR, St. John's, NL, Canada, October 2006.

[11] D. Crisp, The State-of-the-Art in Ship Detection in Synthetic Aperture Radar Imagery, DSTO-RR-0272, Australian Government Department of Defence, Defence Science and Technology Organisation, 2004.

[12] J. Marcum "A statistical theory of target detection by pulsed radar," IRE Transactions on Information Theory, vol. 6 , Iss. 2, 1960 (reprint of RAND Research Memo RM-754, December 1947).

[13] J. Lee, R. Haralick, and L.Shapiro, "Morphologic Edge Detection", IEEE Journal of Robotics and Automation, pp. 142-156, vol. RA-3, no. 2, April 1987.

[14] R. Haralick, and L.Shapiro, Computer and Robot Vision, vol 1, Addison-Wesley, 1992, pp. 28-48.

[15] The Mathworks, Inc., Image Processing Toolbox User's Guide: http://www.mathworks.com/help/images/ref/bwlabel.html.

[16] R. Gonzalez, R Woods, and S. Eddins, Digital Image Processing Using MATLAB, Upper Saddle Rive, NJ, Pearson Prentice Hall, 2004.

[17] C. Pilcher, A Khotanzad, "Maritime ATR using Classifier Combination and High Resolution Range Profiles", Transactions on Aerospace and Electronic Systems Vol. 47, No. 4 Oct. 2011.

[18] IHS Jane's Defense and Security Intelligence and Analysis, https://janes.ihs.com.

[19] R Bellman. Dynamic Programming. Courier Dover Publications, (2003).

[20] USN Signalman Manual Chapter 13, Published by Naval Education and Training Professional Development and Technology Center, NAVSUP Logistics Tracking Number 0504-LP-026-8790, 1995.

[21] R Bellman. Dynamic Programming. Courier Dover Publications, (2003).

[22] S. Kent N. G. Kasapoglu and M. Kartal, "Radar Target Classification Based on Support Vector Machines and High Resolution Range Profiles," Radar Conference, 2008, pp. 1-6. RADAR '08. IEEE.

[23] M.N. Saidi, K. Daoudi, A. Khenchaf, B. Hoeltzener, D. Aboutajdine, "Automatic Target Recognition of Aircraft Models Based on ISAR Images," International Radar Conference - Surveillance for a Safer World, 2009.

[24] T. Kawaharay, S. Todaz, A. Mikamiz, M. Tanabez, "Automatic Ship Recognition Robust Against Aspect Angle Changes and Occlusions," Radar Conference (RADAR), 2012 IEEE.

[25] C. Cortes, V. Vapnik, "Support-Vector Networks", Machine Leaming, Vol 20, pp. 273-297, Kluwer Academic Publishers, Boston, 1995.

[26] MATLAB Release 2011b, The MathWorks, Inc., Natick, Massachusetts, United States. 